\title{
Rapid, low cost and sensitive detection of Calreticulin mutations by a PCR based amplicon length differentiation assay for diagnosis of myeloproliferative neoplasms
}

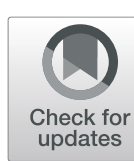

Ngo Tat Trung ${ }^{1,2,3 \dagger}$, Dao Thanh Quyen 2,3, Nghiem Xuan Hoan ${ }^{2,5}$, Dao Phuong Giang ${ }^{2,3}$, Tran Thi Huyen Trang ${ }^{1,2,3}$, Thirumalaisamy P. Velavan ${ }^{2,5}$, Mai Hong Bang ${ }^{2,4+}$ and Le Huu Song ${ }^{2,6^{*}+}$ (D)

\begin{abstract}
Background: Calreticulin (CALR) gene mutations are currently recommended as biomarkers in diagnosis of patients with myeloproliferative neoplasms (MPN) with Jak2 V617F negative phenotype. Our aim was to establish a rapid, low cost and sensitive assay for identification of CALR gene mutations and to validate the diagnostic performance of the established assay in a patient cohort with different clinical MPN phenotypes.

Methods: One hundred five Philadelphia-negative MPN patients, including polycythemia vera (PV), essential thrombocythaemia (ET), and primary myelofibrosis (PMF) were initially screened for JAK2 mutations by amplificationrefractory mutation system (ARMS-PCR) methodology and were further subjected to detection of CALR gene mutations by our in-house assay, a PCR based amplicon length differentiation assay (PCR-ALDA). The PCR-ALDA methodology was compared with real time PCR and Sanger sequencing methods. Furthermore, the analytical sensitivity of the assay was established.

Results: PCR - ALDA approach was able to detect and discriminate the pseudo-positive samples containing more than 1\% CALR mutant alleles. CALR mutations were not detected in 63 Jak2 V617F positive cases in all three methods. In contrast, amongst 42 Jak2 V617F negative cases, both PCR-ALDA and Sanger sequencing coherently identified 12 CALR mutants compared to 10 CALR mutants detected by real-time PCR method.
\end{abstract}

Conclusion: PCR-ALDA can be utilized as an easy-to-use, rapid, low cost and sensitive tool in the detection of CALR mutations in Philadelphia-negative MPN patients.

Keywords: Myeloproliferative neoplasms, JAK2 V617F, CALR mutations

\section{Background}

The myeloproliferative neoplasms (MPN) are clonal disorders of hematopoietic progenitors that includes the classical chronic myeloid leukemia (CML), polycythemia vera (PV), essential thrombocythemia (ET), primary myelofibrosis

\footnotetext{
* Correspondence: lehuusong@108-icid.com

${ }^{\dagger}$ Ngo Tat Trung, Mai Hong Bang and Le Huu Song contributed equally to this work.

${ }^{2}$ Vietnamese - German Center for Medical Research, 108 Institute of Clinical Medical and Pharmaceutical Sciences, No 1, Tran Hung Dao Street, Hai Ba Trung District, Hanoi, Vietnam

${ }^{6}$ Faculty of Tropical and Infectious Diseases, 108 Institute of Clinical Medical and Pharmaceutical Sciences, Hanoi, Vietnam

Full list of author information is available at the end of the article
}

(PMF), chronic eosinophilic leukemia (CEL), chronic myelomonocytic leukemia (CMML), and systemic mastocytosis [1]. Three distinct phenotypes of PV, ET and PMF mainly constitute to Philadelphia-negative myeloproliferative syndromes [2].

Genetic alterations in both Janus Kinase 2 (Jak2) and thrombopoietin receptor myeloproliferative leukemic $(M P L)$ virus oncogenes serve as molecular targets in the diagnosis of MPN [3]. Approximately, 60\% of patients with MPN carry a non-synonymous substitution (V617F) in exon14 of the Jak2 [4]. Among patients with distinct clinical phenotypes, 90\% with PV and 60\% with ET and PMF also carry these discrete gene mutations. Likewise,

(c) The Author(s). 2019 Open Access This article is distributed under the terms of the Creative Commons Attribution 4.0 International License (http://creativecommons.org/licenses/by/4.0/), which permits unrestricted use, distribution, and reproduction in any medium, provided you give appropriate credit to the original author(s) and the source, provide a link to the Creative Commons license, and indicate if changes were made. The Creative Commons Public Domain Dedication waiver (http://creativecommons.org/publicdomain/zero/1.0/) applies to the data made available in this article, unless otherwise stated. 
other Jak2 variants in exon 10 and 12 [4, 5] and non-synonymous MPL variant in exon 10 (W515 L and W515K) [5, 6] were also reported to occur among $5 \%$ of patients with MPN.

Albeit, Jak2/MPL genetic variants essentially contribute in the diagnoses of MPN, a significant number of MPN patients can be missed in genetic screening due to the absence of these mutations. Nevertheless, $80-85 \%$ of Jak2 V617F negative MPN patients can be diagnosed with a recently discovered distinct frame-shift mutation in exon 9 of the calreticulin $(C A L R)$ gene $[7,8]$ which weakens $\mathrm{Ca} 2+$ binding affinity of CALR $[9,10]$. Since its discovery, CALR domain serve as valuable molecular target for the diagnosis of clonal MPNs and since then World Health Organization (WHO) has revised the diagnoses algorithm for patients with Philadelphia-negative MPNs $[1,11]$.

Until date, several CALR mutations had been described. However, two of those namely, type - 1 (52 bp deletion) and a type-2 (5 bp insertion) mutations account for $85 \%$ of Jak2 V617F Philadelphia-double negative MPNs [7, 8]. Until date, consensus has yet not been established for use of a single given methodology for CALR genotyping [12-14]. Current $C A L R$ genetic mutation screening methods include high resolution melting curve analysis (HRMA), direct Sanger sequencing and real-time PCR based techniques that utilize allele specific probes. All current available assays in the detection of CALR mutations are arguable, either on cost, sensitivity and/or specificity.

This study aimed to establish simultaneous detection of CALR type- 1 and CALR type- 2 mutations following a
PCR based method to differentiate the alleles based on amplicon size on a normal agarose gel. This established PCR based amplicon length differentiation assay (PCR-ALDA) was further validated and evaluated with other available assays for their diagnostic performance in a cohort of Vietnamese patients with MPN devoid of JAK2 V617F mutation.

\section{Methods \\ Patient population}

A total 105 Philadelphia-negative MPN patients were recruited at 108 Military Central Hospital from January 2017 until November 2018 and classified based on clinical phenotype as PV $(n=16)$, ET $(n=64)$, and PMF $(n=25)$. The recently revised WHO diagnoses criterion for MPNs was used to define PV, ET and PMF cases [11]. The entire study flow is illustrated in Fig. 1. In brief: All recruited MPN patients were initially screened for $\mathrm{BCR}-\mathrm{ABL}$ transcripts to differentiate BCR-ABL positive or negative MPN patients using real time PCR. All 105 MPN patients $\mathrm{BCR}-\mathrm{ABL}$ transcripts negative were subjected to an ARMS-PCR method to screen for the presence or absence of the Jak2 V617F mutation. Irrespective of the presence or absence of wildtype or mutant, all $105 \mathrm{MPN}$ patients were subjected to an in-house developed PCR based protocol (ALDA -amplicon length differentiation assay) for simultaneous detection of CALR type- 1 and CALR type- 2 mutations. Furthermore the ALDA assay was comparatively evaluated with Sanger sequencing and

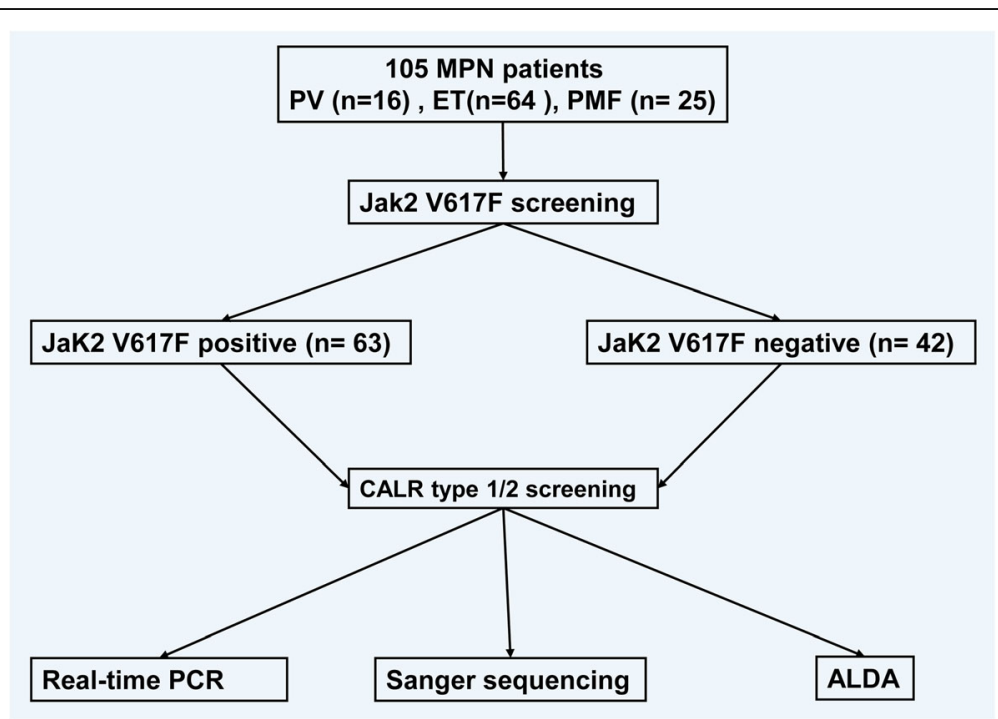

Fig. 1 Study design. All recruited MPN patients were initially screened for BCR-ABL transcripts to differentiate BCR-ABL positive or negative MPN patients using real time PCR. All 105 MPN patients BCR-ABL transcripts negative were subjected to an ARMS-PCR method to screen for the presence or absence of the Jak2 V617F mutation. Irrespective of the presence or absence of wildtype or mutant, all 105 MPN patients were subjected to an in-house developed PCR based protocol (ALDA -amplicon length differentiation assay) for simultaneous detection of CALR type-1 and CALR type-2 mutations. Furthermore the ALDA assay was comparatively evaluated with Sanger sequencing and other available real-time PCR methodologies as described by Zinke et. al [13] 
other available real-time PCR methodologies as described by Zinke et.al [13].

Upon hospital admission, $1 \mathrm{ml}$ of venous blood was collected and stored as $0.5 \mathrm{ml}$ aliquot each until further use. The haematological profile of recruited patients is summarized in Table 1 . One aliquot of $0.5 \mathrm{ml}$ was utilized for RNA extraction, cDNA conversion followed by a real time PCR assay, whereas the other $0.5 \mathrm{ml}$ aliquot of blood for DNA extraction and subsequent genotyping of Jak2 V617F and CALR mutations. The utilized primer pairs in this study are provided in Additional file 1: Table S1.

\section{Screening for $B C R-A B L$ transcripts}

The $B C R-A B L$ transcripts was screened in patients peripheral blood using a standard assay [15]. In brief: $0.5 \mathrm{ml}$ of total blood was mixed with $4.5 \mathrm{ml}$ erythrocyte lysis buffer $(20 \mathrm{mM}$ Tris $\mathrm{HCl} \mathrm{pH} 6.8 ; 5 \mathrm{mM} \mathrm{MgCl} 2 ; 10 \mathrm{mM} \mathrm{NaCl}$ ) and was centrifuged at $1500 \mathrm{rpm}$ for $15 \mathrm{~min}$ at room temperature. The supernatant was discarded and $0.5 \mathrm{ml}$ of TRIzol reagent (ThermoFisher Scientific Inc. Singapore) was suspended to the white blood cell (WBC) pellets. Following the manufacturers protocol, the total RNA was isolated and reconstituted in $50 \mu \mathrm{l}$ diethylpyrocarbonate (DEPC) treated water. Approximately $500 \mathrm{ng}$ of total RNA was reverse transcribed to cDNA using RevertAid First Strand cDNA Synthesis Kit (ThermoFisher Scientific Inc., Singapore), following the manufacturers instruction. Subsequently, the European Leukemia Net (ELN) standard real-time PCR protocol was followed for detecting $B C R-A B L$ transcripts [15].

\section{Screening of Jak2 V617F variant by ARMS-PCR}

The Jak2 V617F mutation was screened following the amplification-refractory mutation system (ARMS-PCR) methodology [16]. In brief: four primer pairs were designed, two were outer primer pairs and two remained as targeted primer pairs that were allele specific. The outer primer pairs are Tr-Jak2-F: 5'- TCCT CAGAACGTTGATGGCAGTTG- $3^{\prime}$ and the TRJak2-R: 5' -TCAGTTTCAAAAATACTTAACTCCTGT $-3^{\prime}$ and the amplification yield a product size of $405 \mathrm{bp}$. Two allele specific primer pairs targeting the mutant and major wild-type allele were also designed namely
Tr-V617F-WT-F: 5'-GCATTTGGTTTTAAATTATGGAGTATTTG and Tr-V617F-MT-R: 5'-GTTTTACTTACTCTCGTCTCCACATAA-3'. The outer Tr-Jak2-F and Tr-V617F-MT-R would generate mutant amplicon of $279 \mathrm{bp}$, whereas the primer pairs Tr-Jak2-R and $\mathrm{Tr}$ V617F-WT-F generate wild type amplicon of $181 \mathrm{bp}$. The locus, primer position and orientation of allele specific primers used in ARMS-PCR is illustrated in Fig. 2. The thermal cycling was with 32 cycles of $15 \mathrm{~s}$ at $95^{\circ} \mathrm{C}$ denaturation; $30 \mathrm{~s}$ at $60^{\circ} \mathrm{C}$ annealing and $30 \mathrm{~s}$ at $72^{\circ} \mathrm{C}$ extension. The presence of mutation V167F yields an amplicon size of $279 \mathrm{bp}$, whereas wild type alleles indicated by an amplicon of $181 \mathrm{bp}$. The utilized ARMS-PCR methodology was further evaluated for their performance using standard/ blinded samples provided by UK-NEQAS agency (http:// www.ukneqasli.co.uk/eqa-pt-programmes/). Additionally, ARMS-PCR amplicons were subsequently subjected to Sanger sequencing to confirm the presence of the mutant or the major wildtype allele [17].

\section{CALR type-1 and -2 genotyping by amplicon length differentiation assay}

A PCR based method was developed for simultaneous detection of CALR type-1 (52 bp deletion) and a type- 2 (5 bp-insertion). This established methodology was a PCR based protocol termed as amplicon length differentiation assay (PCR-ALDA). In brief, two primer pairs were used for this assay. The forward primer was chosen as previously described before in $[7,8]$ as TR-CALR-F: 5'-ACAAATGAAGGACAAACAGGACGA-3', whereas the reverse primer was tailored [18] and was designed by Vector NTI.11.3 (ThermoFisher Scientific Inc. Singapore) using CALR gene sequences as obtained from NCBI database (GenBank Accession Nr. NG_029662) Q-Re-Del-CALR: 5' CGGGGACATCTTCCTCCTCAT-3'. The primers utilized were able to efficiently and unbiasedly amplify both wild-type and known type-1 and -2 CALR mutant alleles. The concentration of individual primers is $0.25 \mathrm{pmol} / \mu \mathrm{l}$. The reaction was run in an Eppendorf Master cycler (Eppendorf, Hamburg, Germany) with 32 cycles of $95^{\circ} \mathrm{C}$ for $15 \mathrm{~s} ; 60^{\circ} \mathrm{C}$ for $30 \mathrm{~s} ; 72{ }^{\circ} \mathrm{C}$ for $30 \mathrm{~s}$. The amplicons were electrophoresed on a $2.5 \%$ agarose gel. A 171 base pair CALR wild-type allele

Table 1 Baseline characteristics of all PMN patients and subgroups classified into ET, PMF and PV syndrome

\begin{tabular}{|c|c|c|c|c|c|}
\hline Clinical characteristics & PMN $(n=105)$ & $\mathrm{ET}(n=67)$ & $P V(n=16)$ & PMF $(n=22)$ & $P^{a}$ \\
\hline Gender - male (\%) & $77(73.3)$ & $50(74)$ & $10(62.5)$ & $17(77.3)$ & NS \\
\hline Age (years) & 64 (16-96) & $64(16-96)$ & $69(29-86)$ & $57(29-85)$ & NS \\
\hline WBC $\left(\times 10^{3} / \mathrm{mL}\right)$ & $14.3(1.9-87.2)$ & $14.8(6.8-66.5)$ & $8.2(5.2-31.9)$ & $15.5(1.9-87.2)$ & 0.039 \\
\hline $\mathrm{RBC}\left(\times 10^{6} / \mathrm{mL}\right)$ & $4.8(2.1-9.7)$ & $4.5(2.5-8.1)$ & $5.7(5.4-9.7)$ & $4.5(2.14-9.7)$ & 0.0002 \\
\hline $\operatorname{PLT}\left(\times 10^{3} / \mathrm{mL}\right)$ & $710(45-2301)$ & $820(376-2301)$ & $233(150-803)$ & $246(45-1776)$ & $6.15 e-11$ \\
\hline $\mathrm{Hb}(\mathrm{g} / \mathrm{L})$ & $138(10.5-211)$ & $132(10.5-188)$ & $185(167-211)$ & $125(66-178)$ & $2.17 \mathrm{e}-08$ \\
\hline
\end{tabular}

Abbreviation: $\mathrm{Hb}$, hemoglobin; PLT, platelets; WBC, white blood cell; RBC, red blood cell; NS, not significant. Values given are medians and range. (a) in comparison between subgroups (ET, PV and PMF). $P$ values were calculated by Chi-squared and Kruskal-Wallis test where appropriate 

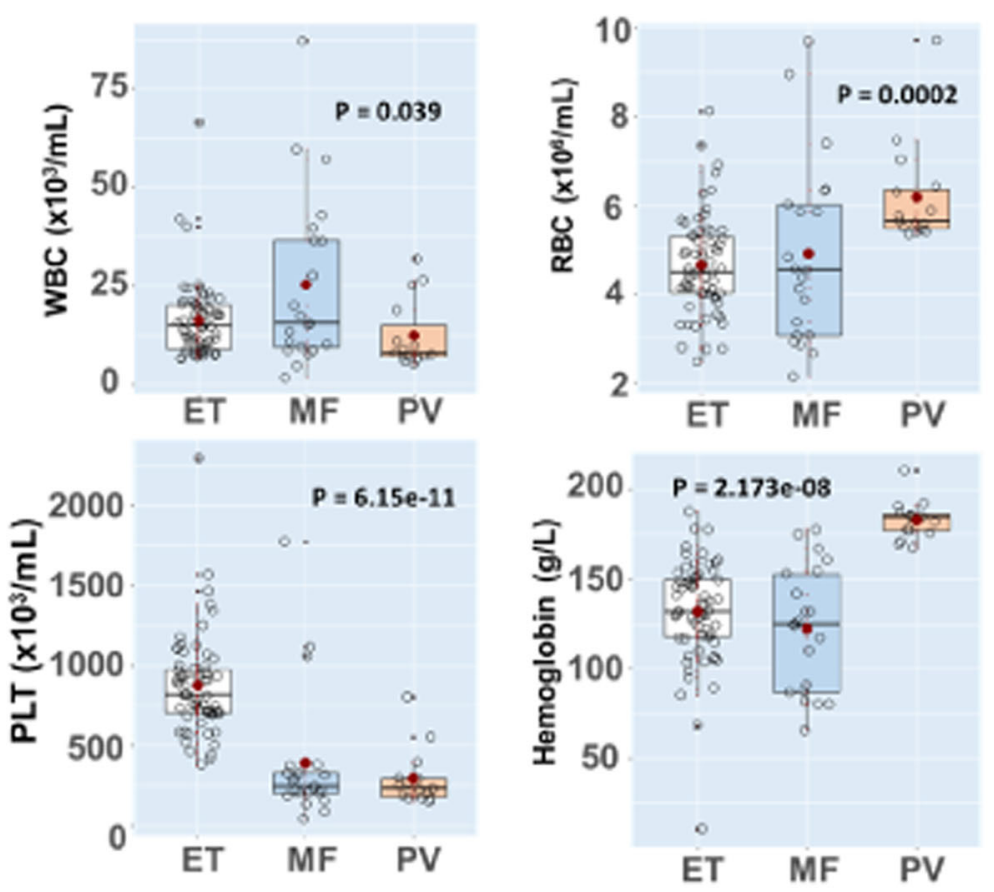

A
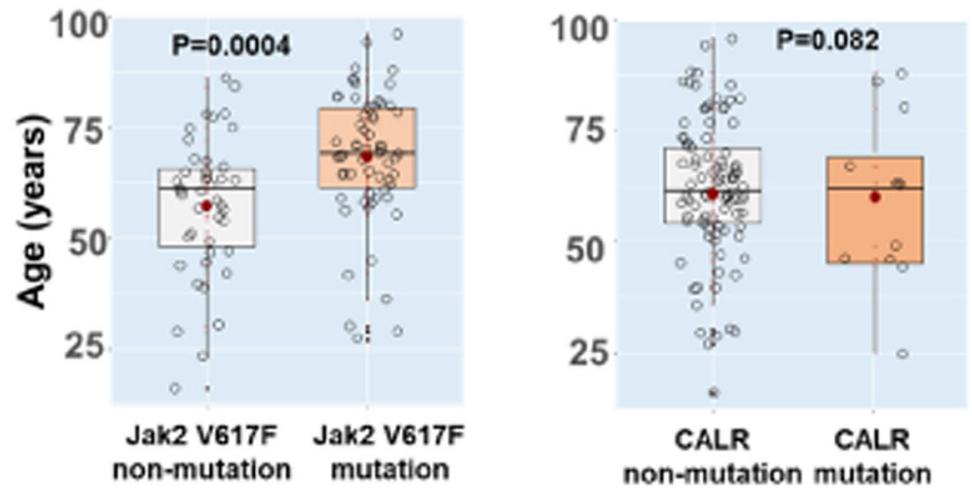

B

Fig. 2 Distribution of haematological values and Jak2 V617F, CALR mutant alleles in different study population. Panel a: Haematological parameters in: ET, PMF and PV subgroups. Panel b: Age distribution and Jak2 V617F and CALR mutations; PLT: platelets; WBC: white blood cell; RBC: red blood cell; PLT: platelet; Hb: haemoglobin

against the deleted or inserted CALR alleles could be visualized on the agarose gel (Fig. 3).

\section{CALR type-1 and -2 genotyping by real time PCR}

Two set of HPLC purified primer pairs as described by Zinke et al. [13] were synthesized (Integrated DNA Technology, IDT Singapore). The primer pairs for type -1 mutation detection were CAL1-F: 5'-ACAGGACGAGGAGCAGATGA-3' and CAL1-R: 5'-GGACATCTTCCTCCTCATCTTCCT-3' that yield a $97 \mathrm{bp}$ product whereas for type-2 mutation, the primer pairs were CAL2-F: 5' - GCTTAAGGAGGAGGAAGAAGACAA-3'; and
CAL2-R: 5'-TGTCCTCATCATCCTCCGACAATT-3', a $79 \mathrm{bp}$ product for the type- 2 mutation. The primer CAL1-F contains a G/T mismatch to further increase specificity, whereas the two fluorescent probe namely CAL1: FAMTGTCCTCCTCATCCTCCTCATCCTCATCT-BHQ1 and CAL2-: FAM- CTGCCTCCTCCTCCTCTTTGCGT-BHQ1 were used to hybridize to the amplified products of the type - 1 deleted CALR or type -2 inserted CALR respectively [13] The real-time PCR assay mixtures consisted of $7.5 \mu \mathrm{l}$ Taqman real-time PCR master Mix (Qiagen, Hilden, Germany), $5 \mu \mathrm{l}$ of genomic DNA or cDNA template, $1 \mathrm{ul}$ of $5 \mathrm{pmol} / \mathrm{ul}$ of primers and $0.5 \mathrm{ul}$ of $5 \mathrm{pmol} / \mathrm{ul}$ probe. The 
A

Tr-V617F-WT-F

Tr-Jak2-F

DNA pol

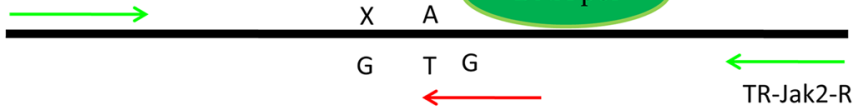

Tr-V617F-MT-R

B
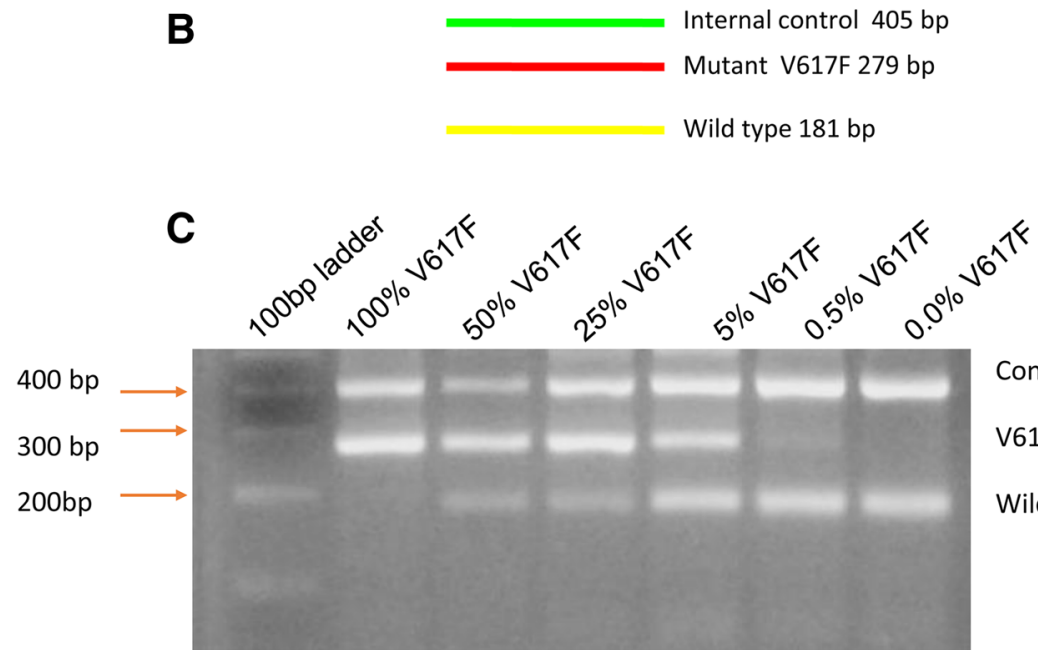

Control $405 \mathrm{bp}$

V617F 279 bp

Wild type $181 \mathrm{bp}$

Fig. 3 Jak2 V617F screening by ARMS-PCR. Panel a with tetra primers: Jak2 V617F mutant allele amplified by primers (Tr-V617F-MT-R/Tr-Jak2-F) resulting in 279 bp product, whereas wild type allele amplified by primer pair (Tr-V617F-WT-F/TR-Jak2-R) resulting in 181 bp product. The amplicon produced by two outer primer pairs (Tr-Jak2-F/TR-Jak2-R) result in 405 bp product (internal control). Panel b Visualization on an agarose gel with dilution series to show resolution of tetra primer PCR product: Upper internal control (405 bp), V617F mutant (279 bp) and the Jak2 wild type (181 bp). Detection is achieved in samples containing up to $0.5 \%$ V617F mutant allele

reactions were run in the Stratagene M3000P device (San Diego, CA, USA) with a pre-incubation step at $50^{\circ} \mathrm{C}$ for 15 $\mathrm{min}$, initial denaturation at $95^{\circ} \mathrm{C}$ for $5 \mathrm{~min}$, followed by $45 \mathrm{cy}$ cles of $95^{\circ} \mathrm{C}$ for $15 \mathrm{~s}$ and $60^{\circ} \mathrm{C}$ for $60 \mathrm{~s}$.

\section{Statistical analysis}

All statistical analyses were performed using the $\mathrm{R}$ version 3.1.2 (http://www.r-project.org). Chi-square test was performed to compare categorical variables. Mann-Whitney $\mathrm{U}$ and Kruskal-Wallis tests were used to compare quantitative variables between two groups where appropriate. Revised WHO diagnostic criteria for MPN were used as reference to determine the diagnostic performance of the in-house procedure [11]. The statistical significance was set to a two-sided $P$ value $<0.05$.

\section{Results}

\section{Baseline characteristics of MPN patients}

Recruited patients were diagnosed based on clinical manifestations and haematological tests and subsequently classified into three groups: PV, ET and PM based on the revised WHO diagnostic criteria [11]. The screening of BCR-ABL transcripts performed to differentiate BCR-ABL positive or negative MPN patients confirmed that all investigated 105 MPN patients were BCR-ABL transcripts negative (Philadelphia chromosome negative). The baseline characteristics and the clinical parameters of the investigated study groups are presented in Table 1 and Fig. 2, respectively. A majority of patients were male (73\%) with a median age of 64 years (range: 16-96). No difference in median age between three subgroups of MPNs (PV, ET and PM) was observed. Around 65 and $76 \%$ of all patients had WBC and Platelet (PLT) level above the upper normal limit (UNL), respectively. Whereas, $17 \%$ of those had increased levels of red blood cell (RBC) above UNL. PLT levels were significantly higher in ET patients than PV and PMF groups $(P<0.0001)$. Compared to ET and PM patients, the levels of Haemoglobin $(\mathrm{Hb})$ and $\mathrm{RBC}$ in PV patients were significantly higher $(\mathrm{P}<0.0001$ and 0.0002 , respectively); In contrast, $\mathrm{WBC}$ levels were lower $(P=$ 0.039). The clinical features of study subjects were further characterized based on the presence of Jak2 V617F and CALR mutations. Jak2 V617F mutations were observed more frequently in older than younger patients $(P=0.0004)$. This finding indicates a possible senescence as a risk factor among these patients. In contrast, no significant contribution of age observed in the distribution 
of CALR mutations among the study groups. To understand effect of these two mutations on contributing to hematological characterizations, we analysed the association of Jak2 V617F and CALR mutations with hematological measurements including WBC, RBC, PLT and $\mathrm{Hb}$ among all 105 patients. No significant association of hematological parameters either with Jak2 V617F or CALR mutation was observed $(P>0.05)$.

\section{Analytical sensitivity of ARMS-PCR and the distribution of Jak2 V617F}

In order to determine the relative concentration at which ARMS-PCR can distinguish mutant allele fragments from wild-type, a stock DNA containing 100\% V617F mutant fragment was diluted against wild type DNA extracted from healthy donor and a dilution series was made from 100, 50, $25,5,0.5,0 \%$ until eventual negativity. All the dilutions were to $100 \mathrm{ng} / \mathrm{ul}$ and $5 \mathrm{ul}$ (equal to $500 \mathrm{ng}$ ) was used as template for each ARMS- PCR. Product mixtures were run through $1.2 \%$ agarose gel to resolve the mutant (297 bp) against wild type (181 bp) or internal control (405 bp). As observed in Fig. 3, ARMS-PCR could detect the presence of V617F mutant allele at a concentration of $0.5 \%$ and the threshold for such a limit of detection (LOD) was repeated several experiments by three independent technicians. Additionally, the external quality control for Jak2 V617F identification was performed on blinded samples provided by the UK-NEQNAS that additionally acquired 100\% accuracy (data not shown).

Subsequently, ARMS-PCR assay was used to screen for V617F, whereas Sanger sequencing was used for the screening of mutations within exon 12 of Jak2. We observed that $42(40 \%)$ were negative for V617F mutant and 63 out of $105(60 \%)$ MPN cases harboured Jak2 V617F mutation. Among those, 60\% (38/64) of ET patients, 44\% (11/25) of PMF patients, and 87.5\% (14/16) of PV patients had this mutation. Nevertheless, we did not detect Jak2 exon 12 mutation in any recruited subjects. Patients with and without Jak2 V617F mutation were then subjected to CALR mutations identification by Zinke's method [13], Sanger sequencing and by established PCR based ALDA assay.

\section{PCR- ALDA and CALR genotyping}

Both homo and heterozygous deletions can well be detected based on amplicon size differentiation. CALR del type- 1 can well be distinguished by a 52 bp deletion with a product size of $119 \mathrm{bp}$ (CALR del type 1 - homozygous mutant), a product size of 171 and $119 \mathrm{bp}$ (CALR del type 1 - heterozygous mutant), whereas CALR type - 2 polymorphism well characterized by an insertion of $5 \mathrm{bp}$ with a product size of $171 \mathrm{bp}$ (CALR type 2 homozygous), a product size of 171 and $176 \mathrm{bp}$ (CALR type 2 heterozygous mutant). In order to evaluate the detection limit of PCR-ALDA assay, we mixed DNA sample containing type -1 or type -2 CALR mutant DNA fragments against wild type DNA samples extracted from healthy donor" and a dilution series of $100 \mathrm{ng} / \mu \mathrm{l}$ containing 50, 25, 5, 0.5 and $0 \%$ CALR mutant alleles was utilized. These serial dilutions were treated as template for gel-based PCRs using one primer pairs that unbiasedly amplified both wild-type and mutant CALR alleles. Subsequently, the reaction mixture was electrophoresed on a $2.5 \%$ agarose gel to resolve the 171 base-pair-CALR-wild-type-allele against the deleted CALR type- 1 or type -2 insertion allele. As illustrated in the Fig. 3, PCR-ALDA approach could discriminate the pseudo-positive samples containing more than $1 \%$ CALR mutant alleles from the wild-type background. The results imply that the PCR-ALDA approach could detect more case carrying CALR mutations than Zinke's Real-time PCR approach which was described as a deeper technical sensitivity at levels of $0.1 \%$ (Fig. 4).

\section{Evaluation of PCR-ALDA compared to sanger sequencing and real-time PCR}

To further evaluate the diagnostic performance of our PCR-ALDA approach, we subjected all 63 Jak2 V617F positive and 42 Jak2 negative DNA samples to PCR-ALDA, Zinke's Real-time PCR and Sanger sequencing (Fig. 5). Our data revealed that no CALR mutant allele was detected in all 63 Jak2 V617F positive DNA samples by these methods. In contrast, amongst 42 Jak2 V617F negative cases, PCR-ALDA and Sanger sequencing equally identified 12 CALR mutant samples (Additional file 2: Figure S1), whereas only 10 mutant samples were detected by Zinke's Real-time PCR (Additional file 3: Figure S2).

\section{Discussion}

Accurate detection of disease-specific mutations facilitates precise diagnostics and better treatment regimens. In the scope of MPN, the best example is the $B C R-A B L$ associated chronic myeloid leukemia $(B C R-A B L$ positive CML) [11]. No other genetic lesions in MPN acquire a similar degree of diagnostic accuracy or therapeutic relevance like that of $B C R-A B L$ positive CML. However, Jak2 mutations are detected in significant portions of Philadelphia-negative MPN patients including PV, ET, PMF and in a minor part of the patients carrying $M P L$ mutations. Therefore, both Jak2 or MPL mutations are used as clonal markers in establishing the diagnosis of MPN $[1,11]$. The fact is that not all MPN patients possess Jak2 mutations and only minor proportion of MPN patients carries $M P L$ mutations, indicating that the diagnostic value of Jak2 or MPL mutations is limited by suboptimal sensitivity and specificity. The molecular diagnostic gap in Jak2/MPL-unmutated MPN patients is 


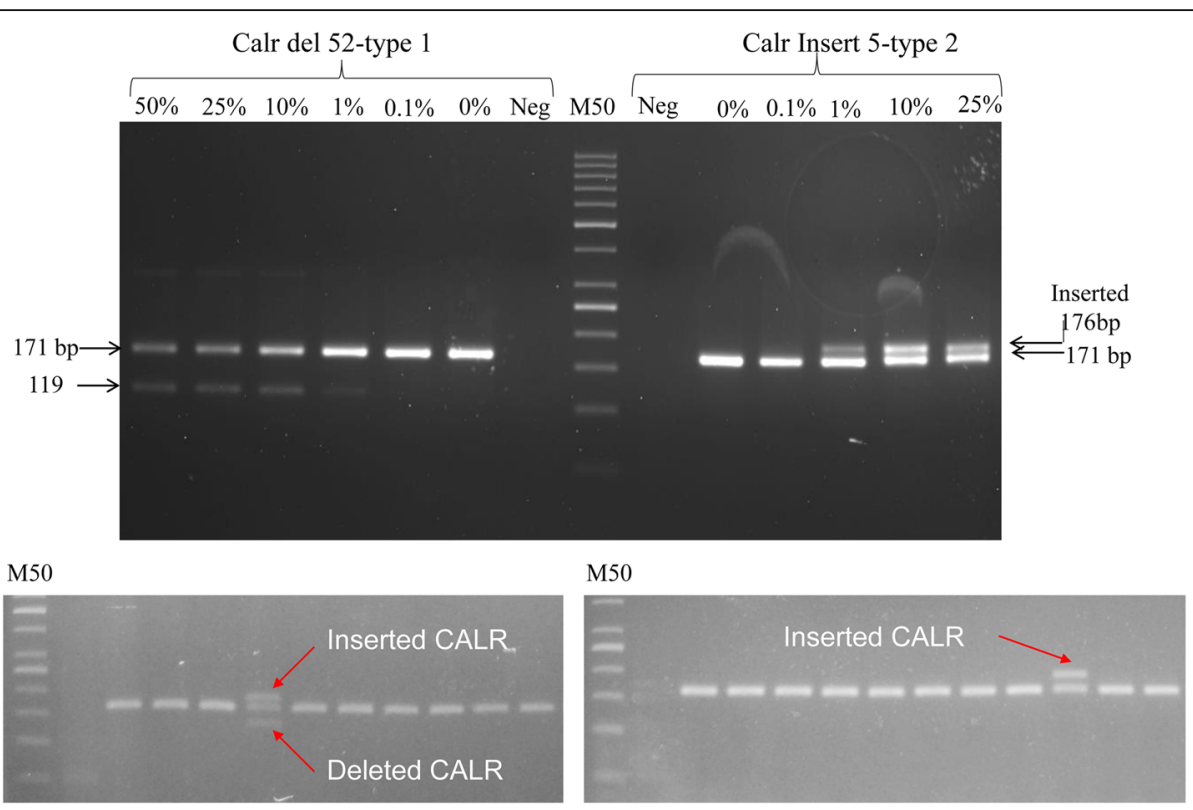

Fig. 4 CALR genotyping and Limit of detection by PCR- ALDA. Dilution series were made by mixing 50\% deleted CALR (upper left panel) or inserted CALR (upper right panel) containing DNA samples against wild type DNA. The dilution series were subjected to PCR-ALDA in which only one primer pair is used to unbiasedly amplify wild-type CALR allele and CALR mutant alleles. Subsequently, the reaction mixture was electrophoresed on a 2.5\% agarose gel. The $171 \mathrm{bp}$-CALR-wild-type-allele against the $119 \mathrm{bp}$ (type 1) $52 \mathrm{bp}$ deleted CALR allele or 5 bp TTGTC inserted type -2 mutation allele or other deleted and inserted alleles. Lower panel- CALR mutations in selected clinical samples: arrows indicate deletions or insertions

complimented by the recent discovery of CALR mutations in the majority of such cases $[7,8]$. However, the described genetic lesions of CALR gene are very heterogenous that make a technical difficulty to gain a consensus approach for routine diagnosis of CALR mutations in clinical settings.

In this study, we used an ARMS-PCR based Jak2 V617F method to screen for the presence or absence of the Jak2 V617F mutation in BCR-ABL transcript negative MPN patients. This assay is very clinically relevant in the context of acquiring an acceptable limit of detection (LOD) at $0.5 \%$, which is comparable to the levels developed by other groups $[19,20]$. At this level of LOD, we are able to identify 63 Jak2 V617F positive cases (60\%) from 105 recruited MPN samples. The distribution of Jak2 mutation reported in this study was expectable as it falls in range similar to that of previously published data $[3,21]$. The identification of Jak2 V617F split our study cohort into two different

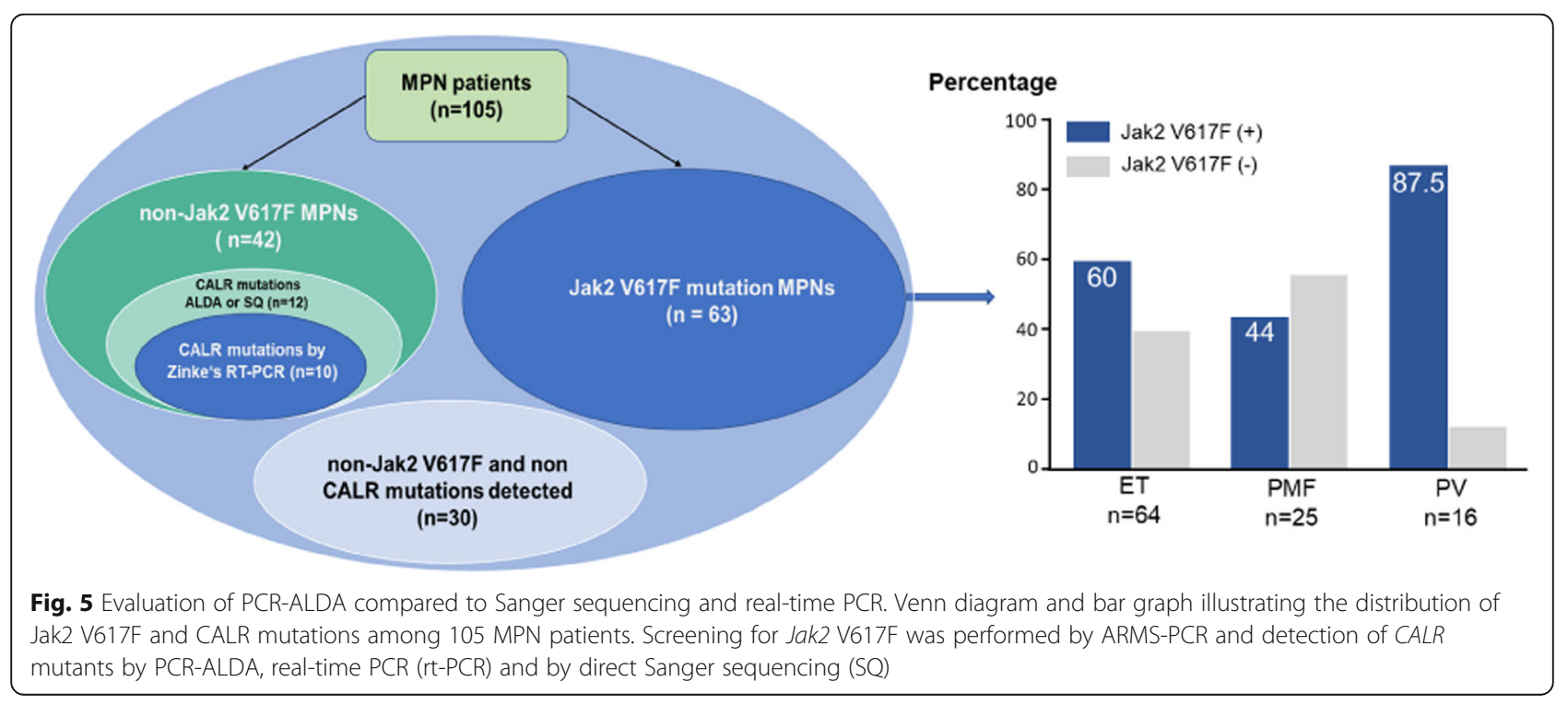


subgroups: Jak2 V617F positive group (Jak-pos) which is found in elder patients and characterized by higher levels of WBC, RBC and PLT but lower level of Hb compared to that of Jak2 V617F negative (Jak-neg) group. This observation is similar to previous studies [22, 23]. Additionally, our Jak2 V617F screening assay works very simple, without special requirement of equipment or personnel. However, we have not yet validated this assay for the purposes of quantitative monitoring like previous studies [19, 20], therefore, we only recommend to use our in-house assay for identification of Jak2 V617F but not for quantification of Jak2 mutant alleles in clinical practice.

As mentioned, the molecular diagnostic gap in JAK2/ $M P L$-unmutated ET/PMF patients can be complemented by the identification of CALR gene mutations $[7,8]$. Therefore, we proposed the PCR-ALDA approach for surveillance of these mutations. Our data revealed that PCR-ALDA approach is obviously acquired a limit of detection (LOD) at $1 \%$. This quantitative level is not stronger than that of Zinkes [13] or the other approach [14]. Nevertheless, as the PCR-ALDA, Zinke's approach and Sanger sequencing were comparatively co-applied to the same studied cohort, the PCR-ALDA assay has demonstrated a better diagnostic performance over that gained by Zinke's approach. PCR-ALDA assay and Sanger sequencing could coherently identify 12 CALR mutant samples but the Zinke's Real-time PCR method detected only 10 positive samples out of Jak2 V617F negative cases. With the single use of Jak2 mutation screening assay (tetra primer assay), only $60 \%(63 / 105)$ of MPN patients were identified, but with the combinatory uses of Jak2 mutation screening and PCR-ALDA assays, $71.4 \%(75 / 105)$ of patients were diagnosed during hospitalization.

We consider that PCR- ALDA assay might not be able to resolve mutant versus the wild-type alleles of $C A L R$ if the mutant amplicons differ at less 5 bps from that of wild-type. However, CALR mutations that possess deletion or insertion stretches shorter than $5 \mathrm{bps}$ are very infrequent [14]. Thus, we believe that it is seldom for our PCR-ALDA to leak the diagnostics of CALR mutations if the clinical samples contain more than $1 \%$ of mutant alleles. We also be aware that it is needed to have methodologies that can precisely quantify the burden of CALR mutant alleles allowing the physicians to indirectly monitor treatment responses to specific therapies like situations in which the quantitative levels of Jak2 V617F allele burden were used as the predictive marker for treatment response in PV patients $[15,24,25]$. Nonetheless, in this current study, the quantification of CALR mutant alleles has not yet validated therefore, we recommend to utilize the assay for identification of CALR mutations but not for quantification of $C A L R$ mutant alleles.

\section{Conclusions}

In this study, we demonstrated a rapid, low cost and sensitive detection of Calreticulin mutations by a PCR based amplicon length differentiation assay for diagnosis of myeloproliferative neoplasms.

\section{Additional files}

Additional file 1: Table S1. Primer pairs utilized in this study. (DOCX 14 kb)

Additional file 2: Figure S1. Electropherograms illustrating /CALR/ -type1 and -type 2 mutations. (PNG 1375 kb)

Additional file 3: Figure S2. Limit of detection of /CALR/ -type 1 and -type 2 mutants by Zinke's Real-time PCR. (PNG 2205 kb)

\section{Abbreviations}

ALDA: Amplicon Length Differentiation Assay; ARMS: Amplification-refractory mutation system; CALR: Calreticulin; CML: Chronic myeloid leukemia; ET: Essential thombocythemia; Jak2: Janus Kinase 2; MPL: Myeloproliferative leukemic; MPN: Myeloproliferative neoplasms; PM: Primary myelofibrosis; $\mathrm{PV}$ : Polycythemia vera

\section{Acknowledgements}

Authors would like to thank the staff and all participants involved in this study.

\section{Funding}

The authors acknowledge the funding from the Vietnamese Ministry of Science and Technology under grant number KC10.13.16-20 for Dr. Le Huu Song. The funding agency has no role in study design, data collection and analysis, decision to publish, and/or preparation of the manuscript.

\section{Availability of data and materials}

Data and all information enclosed within this article could not be shared due to patient confidentiality.

\section{Authors' contributions}

NTT, LHS and MHB designed and supervised the studies. DTQ, DPG conducted the experiments, documented the clinical data. NTT, NXH analysed the data and interpreted the results. NTT, LHS, NXH, TPV wrote the manuscript. TTHT revised the manuscript. All authors read and approved the manuscript.

Ethics approval and consent to participate

The study was approved by the institutional review board and the ethics committee of the 108 Military Central Hospital (IRB: 108 MCH -KC10.13.16-20). Informed written consent was obtained from all patients and/or from their parents if the participant was below 18 years of age.

Consent for publication

Not applicable.

Competing interests

The authors declare that they have no competing interests.

\section{Publisher's Note}

Springer Nature remains neutral with regard to jurisdictional claims in published maps and institutional affiliations.

\section{Author details}

${ }^{1}$ Centre for Genetic Consultation and Cancer Screening, 108 Institute of Clinical Medical and Pharmaceutical Sciences, Hanoi, Vietnam. ${ }^{2}$ Vietnamese German Center for Medical Research, 108 Institute of Clinical Medical and Pharmaceutical Sciences, No 1, Tran Hung Dao Street, Hai Ba Trung District, Hanoi, Vietnam. ${ }^{3}$ Department of Molecular Biology, 108 Institute of Clinical Medical and Pharmaceutical Sciences, Hanoi, Vietnam. ${ }^{4}$ Faculty of Gastroenterology, 108 Institute of Clinical Medical and Pharmaceutical Sciences, Hanoi, Vietnam. ${ }^{5}$ Institute of Tropical Medicine, Universitätsklinikum Tübingen, Tübingen, Germany. ${ }^{6}$ Faculty of Tropical and Infectious Diseases, 
108 Institute of Clinical Medical and Pharmaceutical Sciences, Hanoi, Vietnam.

Received: 26 February 2019 Accepted: 3 May 2019

Published online: 27 June 2019

\section{References}

1. Barbui T, Thiele J, Gisslinger H, Kvasnicka HM, Vannucchi AM, Guglielmelli P, Orazi A, Tefferi A. The 2016 WHO classification and diagnostic criteria for myeloproliferative neoplasms: document summary and in-depth discussion. Blood cancer journal. 2018:8(2):15.

2. Levine RL, Pardanani A, Tefferi A, Gilliland DG. Role of JAK2 in the pathogenesis and therapy of myeloproliferative disorders. Nat Rev Cancer. 2007;7(9):673-83.

3. Barbui T, Barosi G, Birgegard G, Cervantes F, Finazzi G, Griesshammer M, Harrison C, Hasselbalch HC, Hehlmann R, Hoffman R, et al. Philadelphianegative classical myeloproliferative neoplasms: critical concepts and management recommendations from European LeukemiaNet. Journal of clinical oncology : official journal of the American Society of Clinical Oncology. 2011;29(6):761-70.

4. Kralovics RPF, Buser AS, Teo SS, Tiedt R, Passweg JR, Tichelli A, Cazzola M, Skoda RC. A gain-of-function mutation of JAK2 in myeloproliferative disorders. N Engl J Med. 2005;28:352.

5. Pardanani AD, Levine RL, Lasho T, Pikman Y, Mesa RA, Wadleigh M, Steensma DP, Elliott MA, Wolanskyj AP, Hogan WJ, et al. MPL515 mutations in myeloproliferative and other myeloid disorders: a study of 1182 patients. Blood. 2006;108(10):3472-6.

6. Pikman Y, Lee BH, Mercher T, McDowell E, Ebert BL, Gozo M, Cuker A, Wernig G, Moore S, Galinsky I, et al. MPLW515L is a novel somatic activating mutation in myelofibrosis with myeloid metaplasia. PLoS Med. 2006;3(7):e270.

7. Klampfl T, Gisslinger $H$, Harutyunyan AS, Nivarthi H, Rumi E, Milosevic JD, Them NC, Berg T, Gisslinger B, Pietra D et al. Somatic mutations of calreticulin in myeloproliferative neoplasms. N Engl J Med. 2013;369(25): 2379-90.

8. Nangalia J, Massie CE, Baxter EJ, Nice FL, Gundem G, Wedge DC, Avezov E, Li J, Kollmann K, Kent DG et al. Somatic CALR mutations in myeloproliferative neoplasms with nonmutated JAK2. N Engl J Med. 2013: 369(25):2391-2405.

9. Elf S, Abdelfattah NS, Chen E, Perales-Paton J, Rosen EA, Ko A, Peisker F, Florescu N, Giannini S, Wolach O, et al. Mutant Calreticulin requires both its mutant C-terminus and the Thrombopoietin receptor for oncogenic transformation. Cancer discovery. 2016;6(4):368-81.

10. Pietra D, Rumi E, Ferretti W, Di Buduo CA, Milanesi C, Cavalloni C, Sant'Antonio E, Abbonante V, Moccia F, Casetti IC, et al. Differential clinical effects of different mutation subtypes in CALR-mutant myeloproliferative neoplasms. Leukemia. 2016;30(2):431-8.

11. Tefferi A, Thiele J, Vannucchi AM, Barbui T. An overview on CALR and CSF3R mutations and a proposal for revision of WHO diagnostic criteria for myeloproliferative neoplasms. Leukemia. 2014;28(7):1407-1413.

12. Ji-Hye Park MS, Selim Ramla, Aurélie Truffot and et al. Calreticulin Mutations in Myeloproliferative Neoplasms: Comparison of Three Diagnostic Methods. PloS one. 2015;10(10).

13. Zinke M, Nageswaran V, Reinhardt R, Burmeister T. Rapid and sensitive detection of calreticulin type 1 and 2 mutations by real-time quantitative. PCR. Mol Diagn Ther. 2015;19(5):329-34.

14. Chi J, Manoloukos M, Pierides C, Nicolaidou V, Nicolaou K, Kleopa M, Vassiliou G, Costeas P. Calreticulin mutations in myeloproliferative neoplasms and new methodology for their detection and monitoring. Ann Hematol. 2015;94(3):399-408.

15. Ferdowsi S, Ghaffari SH, Amirizadeh N, Azarkeivan A, Atarodi K, Faranoush M, Toogeh G, Shirkoohi R, Vaezi M, Maghsoodlu M et al. JAK2V617F Allele Burden Measurement in Peripheral Blood of Iranian Patients with Myeloproliferative Neoplasms and Effect of Hydroxyurea on JAK2V617F Allele Burden. Int J Hematol Oncol Stem Cell Res. 2016;10(2):70-8.

16. Little S. Amplification-refractory mutation system (ARMS) analysis of point mutations. Curr Protoc Hum Genet. 2001; Chapter 9:Unit 98.

17. Scott LM, Tong W, Levine RL, Scott MA, Beer PA, Stratton MR, Futreal PA Erber WN, McMullin MF, Harrison CN et al. JAK2 exon 12 mutations in polycythemia vera and idiopathic erythrocytosis. N Engl J Med. 2007;356(5): 459-68.
18. Fu R, Xuan M, Zhou Y, Sun T, Bai J, Cao Z, Zhang L, Li H, Zhang D, Zhang X et al. Analysis of calreticulin mutations in Chinese patients with essential thrombocythemia: clinical implications in diagnosis, prognosis and treatment. Leukemia. 2014;28(9):1912-14.

19. Wu Z, Yuan H, Zhang X, Liu W, Xu J, Zhang W, Guan M. Development and inter-laboratory validation of unlabeled probe melting curve analysis for detection of JAK2 V617F mutation in polycythemia vera. PloS one. 2011; 6(10):e26534.

20. V Jovanovic Al, AM Vannucchi, E Lippert and et al. Establishing optimal quantitative-polymerase chain reaction assays for routine diagnosis and tracking of minimal residual disease in JAK2-V617F-associated myeloproliferative neoplasms: a joint European LeukemiaNet/MPN\&MPNrEuroNet (COST action BM0902) study. Leukemia. 2013;27:2032-39.

21. Tefferi A. Novel mutations and their functional and clinical relevance in myeloproliferative neoplasms: JAK2, MPL, TET2, ASXL1, CBL, IDH and IKZF1. Leukemia. 2010;24(6):1128-38.

22. Duletic AN, Dekanic A, Hadzisejdic I, Kusen I, Matusan-llijas K, Grohovac D, Grahovac B, Jonjic N. JAK2-v617F mutation is associated with clinical and laboratory features of myeloproliferative neoplasms. Coll Antropol. 2012; 36(3):859-65.

23. Karkucak M, Yakut T, Ozkocaman V, Ozkalemkas F, Ali R, Bayram M, Gorukmez O, Ocakoglu G: Evaluation of the JAK2-V617F gene mutation in Turkish patients with essential thrombocythemia and polycythemia vera. Mol Biol Rep. 2012;39(9):8663-67.

24. Passamonti F, Rumi E. Clinical relevance of JAK2 (V617F) mutant allele burden. Haematol. 2009;94(1):7-10.

25. Vannucchi AM, Pieri L, Guglielmelli P. JAK2 Allele Burden in the Myeloproliferative Neoplasms: Effects on Phenotype, Prognosis and Change with Treatment. Ther Adv Hematol. 2011;2(1):21-32.

\section{Ready to submit your research? Choose BMC and benefit from:}

- fast, convenient online submission

- thorough peer review by experienced researchers in your field

- rapid publication on acceptance

- support for research data, including large and complex data types

- gold Open Access which fosters wider collaboration and increased citations

- maximum visibility for your research: over $100 \mathrm{M}$ website views per year

At BMC, research is always in progress.

Learn more biomedcentral.com/submissions 